\title{
A novel surrogate index for hepatic insulin resistance
}

\author{
J. Vangipurapu • A. Stančáková • T. Kuulasmaa • \\ J. Paananen • J. Kuusisto • the EGIR-RISC Study Group • \\ E. Ferrannini • M. Laakso
}

Received: 12 August 2010 / Accepted: 20 October 2010 /Published online: 25 November 2010

(C) Springer-Verlag 2010

\begin{abstract}
Aims/hypothesis In epidemiological and genetic studies surrogate indices are needed to investigate insulin resistance in different insulin-sensitive tissues. Our objective was to develop a surrogate index for hepatic insulin resistance.

Methods A sample of 368 non-diabetic participants (age $43.0 \pm 8.2$ years, BMI $26.0 \pm 4.0 \mathrm{~kg} / \mathrm{m}^{2}$; mean $\pm \mathrm{SD}$ ) whose endogenous glucose production (EGP) was measured with [6- $\left.6^{2} \mathrm{H}_{2}\right]$ glucose in the fasting state and during the euglycaemic-hyperinsulinaemic clamp were included in the study. EGP multiplied by fasting plasma insulin (FPI) concentration was the reference measurement for liver insulin resistance (liver IR). Liver IR index was calculated with linear regression analysis including age, obesity indices, lipids, lipoproteins and several variables regulating glucose metabolism.

Results The following variables were significantly associated with liver IR in multiple forward stepwise regression analysis: insulin AUC in an OGTT, fat mass, HDL-
\end{abstract}

J. Vangipurapu and A. Stančáková contributed equally to this study.

Electronic supplementary material The online version of this article (doi:10.1007/s00125-010-1966-7) contains a complete list of the investigators of the EGIR-RISC Study Group, which is available to authorised users.

J. Vangipurapu • A. Stančáková · T. Kuulasmaa · J. Paananen ·

J. Kuusisto $\cdot$ M. Laakso $(\bowtie)$

Department of Medicine,

University of Eastern Finland and Kuopio University Hospital,

70210, Kuopio, Finland

e-mail: markku.laakso@kuh.fi

E. Ferrannini

Department of Internal Medicine,

University of Pisa School of Medicine,

Pisa, Italy cholesterol and BMI. Liver IR index correlated significantly with EGP $\times$ FPI $(r=0.65, p<0.001)$. In participants with abnormal glucose tolerance, the correlation of liver IR with EGP $\times$ FPI was slightly stronger $(r=0.69, p<$ $0.001)$ than in those with normal glucose tolerance $(r=0.62$, $p<0.001$ ).

Conclusions/interpretation We generated a novel surrogate index for liver insulin resistance correlating strongly with $\mathrm{EGP} \times \mathrm{FPI}$.

Keywords Endogenous glucose production.

Fasting plasma insulin - Liver insulin resistance .

Surrogate marker

$\begin{array}{ll}\text { Abbreviations } \\ \text { EGP } & \text { Endogenous glucose production } \\ \text { FPI } & \text { Fasting plasma insulin } \\ \text { Liver IR } & \text { Liver insulin resistance } \\ \text { RISC } & \begin{array}{l}\text { Relationship between insulin sensitivity and } \\ \text { cardiovascular disease }\end{array}\end{array}$

\section{Introduction}

Insulin resistance and beta cell failure represent the core pathophysiological defects in type 2 diabetes [1]. Insulin resistance, which is also observed in non-diabetic patients, clusters with other metabolic and cardiovascular risk factors, occurring in multiple insulin-sensitive tissues, including skeletal muscle, liver and adipose tissue [1].

The liver plays an important role in the development of hyperglycaemia and dyslipidaemia. Insulin resistance in liver is manifested by overproduction of glucose in the basal state despite the presence of fasting hyperinsulinaemia, 
indicating impaired suppression of hepatic glucose production in response to insulin [2]. Hepatic insulin resistance contributes to fasting and postprandial hyperglycaemia in prediabetic and type 2 diabetic individuals [3, 4]. Thus, elevated endogenous glucose production (EGP) in the presence of high fasting plasma insulin (FPI) concentration suggests hepatic insulin resistance. Since the majority of EGP is known to occur in the liver, EGP $\times$ FPI is considered to be the reference measure for hepatic insulin resistance [5]. The assessment of hepatic insulin resistance by clamp combined with tracer techniques is laborious and expensive, and therefore not feasible for large epidemiological and genetic studies.

Surrogate markers of insulin resistance are widely used in population-based studies. Only one previous surrogate index for hepatic insulin resistance has been published. The study, by Abdul Ghani et al. [6], included 155 MexicanAmerican participants (100 with normal glucose tolerance, 55 with impaired glucose tolerance) who were mostly obese (mean BMI $30.4 \mathrm{~kg} / \mathrm{m}^{2}$ ). Their surrogate index was calculated as the product of insulin and glucose AUCs during the first $30 \mathrm{~min}$ of an OGTT, and it had a correlation of 0.64 with $\mathrm{EGP} \times \mathrm{FPI}$.

We aimed to generate a new surrogate liver insulin resistance (liver IR) index, which would include not only variables from an OGTT, but also various metabolic traits/ markers observed in hepatic insulin resistance. To this end, we validated our index in a large sample of healthy nondiabetic white Europeans [7].

\section{Methods}

Study participants A sample of 368 clinically healthy nondiabetic men $(n=183)$ and women $(n=185)$ from the Relationship between Insulin Sensitivity and Cardiovascular

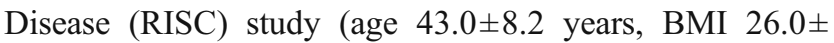
$4.0 \mathrm{~kg} / \mathrm{m}^{2}$; mean \pm SD) with complete data was used to develop and validate our surrogate index of liver IR. In this sample, 288 participants had normal glucose tolerance and 80 had either impaired fasting glucose, impaired glucose tolerance or both [8]. All participants were recruited from 19 research centres in 14 European countries. The protocol was approved by the local Ethics Committees and all participants gave written informed consent. The inclusion of study participants and the study protocol have been described previously [7].

EGP EGP was measured with $\left[6-6^{2} \mathrm{H}_{2}\right]$ glucose in the fasting state and during the euglycaemic-hyperinsulinaemic clamp. A primed continuous infusion of labelled glucose was continued for $2 \mathrm{~h}$ and blood samples obtained during the last $20 \mathrm{~min}$ of the equilibration period to measure the plasma tracer concentration. EGP during the clamp was measured assuming a monocompartment model for glucose kinetics. Insulin sensitivity was measured by the euglycaemic insulin clamp technique [5], using a primed infusion rate of $1 \mathrm{mU} \min ^{-1} \mathrm{~kg}^{-1}$, followed by an exogenous glucose infusion.

OGTT A 2 h OGTT (75 g glucose) was performed after an overnight fast and samples for plasma glucose, insulin and NEFA were drawn.

Laboratory measurements Plasma glucose was measured by the glucose oxidase method. Plasma insulin concentrations were measured by radioimmunoassay. Body composition was determined by bioelectrical impedance (TBF-300 Bioimpedance Balance; Tanita International Division, Middlesex, UK). Total plasma adiponectin was measured using a validated in-house time-resolved immunofluorimetric assay. Glucagon was measured by a glucagon assay (developed in J. Holst's laboratory, University of Copenhagen, Denmark) and NEFA by spectrophotometry. Plasma HDL-cholesterol, triacylglycerol and alanine aminotransferase were measured by standard methods.

Calculations The trapezoidal method was used to calculate glucose and insulin AUC in an OGTT based on 0, 30 and $120 \mathrm{~min}$ data (AUC 0-120 min). The hepatic insulin resistance index proposed by Abdul-Ghani et al. [6] was calculated as described.

Statistical analysis Pearson correlation analysis was performed between EGP $\times$ FPI and all clinical and laboratory variables such as glucose and insulin AUC from an OGTT, fasting NEFA, lipoproteins, measures of adiposity, alanine transaminase, adiponectin and glucagon. Forward multiple stepwise regression analysis was applied to identify the statistically significant determinants of EGP $\times$ FPI. All variables were log-transformed due to their skewed distribution, except for liver IR index, which was normally distributed. The effect of sex and obesity on liver IR index was tested using a univariate linear regression model.

\section{Results}

Liver IR index Variables with a correlation $(r)$ of $r>0.2$ with EGP $\times$ FPI, including BMI, waist, fat mass (\%), HDLcholesterol, total triacylglycerol, adiponectin, glucose AUC 0-120 min and insulin AUC 0-120 min, were included in multiple stepwise linear regression analysis (Table 1). Four 
Table 1 Pearson correlations of clinical and laboratory measurements with hepatic insulin resistance, defined by the product of EGP and FPI

\begin{tabular}{ll}
\hline Variable & $r$ \\
\hline Age & 0.027 \\
Waist & $0.311^{* * *}$ \\
Fat mass & $0.410^{* * *}$ \\
BMI & $0.454^{* * *}$ \\
NEFA & 0.078 \\
HDL-cholesterol & $-0.299^{* * *}$ \\
Triacylglycerol & $0.306^{* * *}$ \\
Alanine transaminase & $0.193^{* * *}$ \\
Adiponectin & $-0.218^{* * *}$ \\
Glucagon & 0.080 \\
Glucose AUC $0-120 \mathrm{~min}$ & $0.236^{* * *}$ \\
Insulin AUC $0-120 \mathrm{~min}$ & $0.562^{* * *}$ \\
\hline
\end{tabular}

${ }^{*} p<0.001$

of these variables remained statistically significant in the regression model, giving the following formula for liver IR index:

Liver IR index $=$

$$
\begin{aligned}
& -0.091+(\log \text { insulin AUC } 0-120 \mathrm{~min} \times 0.400) \\
& +(\log \text { fat mass } \% \times 0.346)-(\log \text { HDL-cholesterol } \times 0.408) \\
& +(\log \text { BMI } \times 0.435)
\end{aligned}
$$

Liver IR index correlated significantly with $\mathrm{EGP} \times \mathrm{FPI}$ $(r=0.65, p<0.001$; Fig. 1$)$ and exhibited a slightly stronger correlation with EGP $\times$ FPI in glucose-intolerant $(r=0.69)$ than in normoglycaemic participants $(r=0.62)$. Liver IR index performed somewhat better in obese participants (BMI $\left.>25 \mathrm{~kg} / \mathrm{m}^{2}, n=206, r=0.62, p<0.001\right)$ than in lean participants (BMI $\leq 25 \mathrm{~kg} / \mathrm{m}^{2}, n=162, r=0.53, p<0.001$ ). The RISC study included men and women. We therefore tested whether sex had any influence on the liver IR index. The correlation coefficients of the liver IR index with EGP $\times$ FPI were similar in both sexes $(r=0.65)$.

We repeated our calculations by replacing glucose and insulin AUC 0-120 min by their ratio (insulin AUC 0120 min: glucose AUC 0-120 min). With this ratio, the model identified the same variables in the regression equation as did the liver IR index, but the correlation with EGP $\times$ FPI was not better $(r=0.64)$. When we added EGP measurement during the clamp to basal EGP (i.e. fasting EGP + clamp EGP), the liver IR index correlated significantly but less strongly $(r=$ $0.59, p<0.001$ ) with (fasting EGP + clamp EGP) $\times$ FPI.

The hepatic insulin resistance index proposed by AbdulGhani et al. [6] had a lower correlation of $r=0.58(p<$ 0.001 ) with $\mathrm{EGP} \times \mathrm{FPI}$ in our study. The correlation was somewhat weaker in participants with normoglycaemia $(r=$
$0.56, p<0.001)$ than in those with abnormal glucose tolerance $(r=0.59, p<0.001)$.

\section{Discussion}

We developed a novel surrogate marker of hepatic insulin resistance. It takes into consideration not only OGTTderived measures of glucose and insulin, but also various metabolic traits related to hepatic insulin resistance, e.g. BMI, fat mass and HDL-cholesterol. Obesity is a known risk factor for hepatic insulin resistance; moreover, intraabdominal visceral fat and abdominal subcutaneous fat also correlate positively with hepatic insulin resistance [9]. Hepatic insulin resistance also reduces HDL-cholesterol level [10].

Hepatic insulin sensitivity determines EGP, which is the main regulator of glucose level in the fasting state. In individuals with abnormal glucose tolerance, and especially in individuals with type 2 diabetes, suppression of EGP is inadequate and contributes to fasting and postprandial hyperglycaemia [3, 4]. Since insulin inhibits EGP, increments in insulin concentrations during an OGTT or clamp exert an inhibitory effect on EGP. We further examined our liver IR index by adding EGP measurement during the clamp to basal EGP, but this did not alter the correlation much, indicating that our index seems to be a good marker for liver IR not only in the fasting, but also in the insulinstimulated state.

In the study by Abdul-Ghani et al. [6], the correlation of the hepatic insulin resistance index, calculated as a product of glucose and insulin AUCs during 0-30 min,

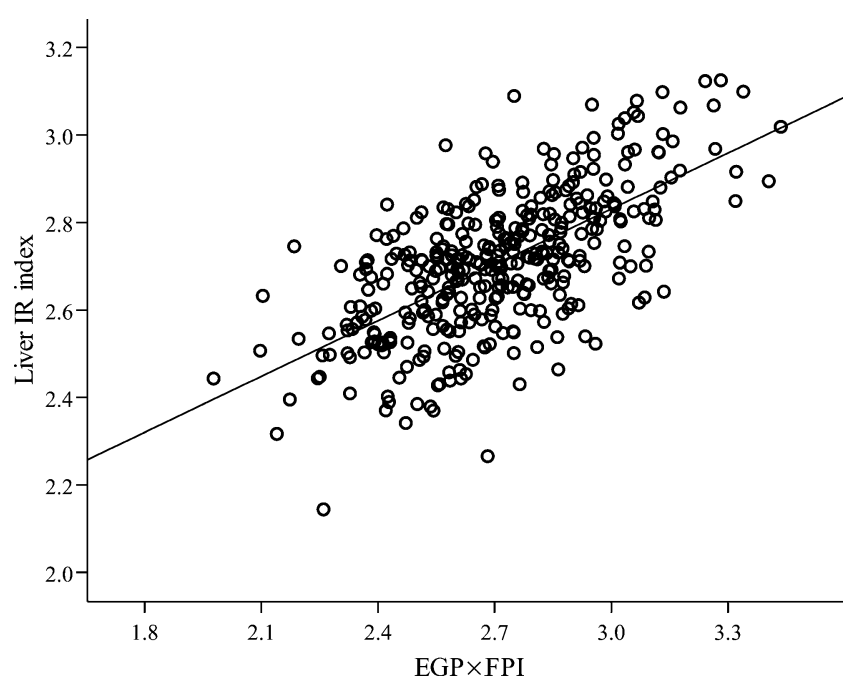

Fig. 1 Pearson correlation of liver IR index as a function of EGP multiplied by FPI $(r=0.65, p<0.001)$. Circles indicate the participants and the diagonal indicates linear regression line 
with EGP $\times$ FPI, was $0.64(n=155)$. In the RISC study, however, which included more than twice as many participants than that cited, the correlation was somewhat weaker $(r=0.58, p<0.001)$. The differences between these results may be due to the smaller sample size in the study by Abdul-Ghani et al. [6] and the larger number of obese participants in their study. Our liver IR index correlated more strongly with EGP $\times$ FPI in obese participants $(r=$ $0.62)$ than in lean participants $(r=0.53)$, which was also reported for the index proposed by Abdul-Ghani et al. [6], where the difference was larger $(r=0.65$ and $r=0.31$ respectively). Therefore, our liver IR index is more suitable for studies including normal-weight and obese participants.

The strength of our study is the availability of gold standard measurements to estimate hepatic insulin resistance (tracers, clamp) in a large European cohort. Furthermore, our liver IR index takes into account clinical and metabolic traits that are characteristic features of hepatic insulin resistance. Nevertheless, our liver IR index will need to be validated in other studies.

In conclusion, we developed a novel surrogate index for hepatic insulin resistance and provided evidence that this index performs well in non-diabetic individuals independently of glucose tolerance and obesity.

Acknowledgements This study was supported by grants from the Academy of Finland (Contract 124243), The Finnish Heart Foundation, The Finnish Diabetes Research Foundation, TEKES (Contract 1510/31/06), EVO grant (Contracts 5232 and 5263) and the Commission of the European Community (Contract LSHM-CT2004_512013 EUGENE2). J. Vangipurapu was supported by a grant from the North Savo Regional Fund.
Duality of interest The authors declare that there is no duality of interest associated with this manuscript.

\section{References}

1. DeFronzo RA (2009) Banting Lecture. From the triumvirate to the ominous octet: a new paradigm for the treatment of type 2 diabetes. Diabetes 58:773-795

2. DeFronzo RA, Ferrannini E, Simonson DC (1989) Fasting hyperglycemia in non-insulin dependent diabetes mellitus: contributions of excessive hepatic glucose production and impaired tissue glucose uptake. Metabolism 38:387-395

3. Weyer C, Bogardus C, Pratley RE (1999) Metabolic characteristics of individuals with impaired fasting glucose and/or impaired glucose tolerance. Diabetes 48:2197-2203

4. Firth RG, Bell PM, Marsh HM, Hansen I, Rizza RA (1986) Postprandial hyperglycemia in patients with noninsulin-dependent diabetes mellitus. Role of hepatic and extrahepatic tissues. J Clin Invest 77:1525-1532

5. DeFronzo RA, Tobin JD, Andres R (1979) Glucose clamp technique: a method for quantifying insulin secretion and resistance. Am J Physiol 237:E214-E223

6. Abdul-Ghani MA, Matsuda M, Balas B, DeFronzo RA (2007) Muscle and liver insulin resistance indexes derived from the oral glucose tolerance test. Diab Care 30:89-94

7. Hills SA, Balkau B, Coppack SW et al (2004) The EGIR-RISC STUDY (the European group for the study of insulin resistance: relationship between insulin sensitivity and cardiovascular disease risk): I. Methodology and objectives. Diabetologia 47:566-570

8. American Diabetes Association (2006) Diagnosis and classification of diabetes mellitus. Diab Care 29(Suppl 1):S43-S48

9. Miyazaki Y, Glass L, Triplitt C, Wajcberg E, Mandarino LJ, DeFronzo RA (2002) Abdominal fat distribution and peripheral and hepatic insulin resistance in type 2 diabetes mellitus. Am J Physiol Endocrinol Metab 283:E1135-E1143

10. Biddinger SB, Hernandez-Ono A, Rask-Madsen C et al (2008) Hepatic insulin resistance is sufficient to produce dyslipidemia and susceptibility to atherosclerosis. Cell Metab 7:125-134 